\title{
Hydrogels with self-assembling ordered structures and their functions
}

\author{
Zi Liang Wu' and Jian Ping Gong ${ }^{2 *}$ \\ Hokkaido University, Japan
}

Biological soft tissue and synthesized hydrogel both belong to the same 'soft and wet' class of substances, that is, they are both soft and contain water. However, whereas biological tissue such as muscles displays various forms of functionality, hydrogels generally have poor functionality, primarily due to the difference in structure-biological tissue has a sophisticated structure whereas most hydrogels are amorphous. In recent years, biomacromolecules, block copolymers and liquid-crystalline molecules have been used to develop self-assembled architectures in synthetic physical or chemical hydrogels via intermolecular noncovalent interactions such as ionic bonding, hydrophobic interactions and hydrogen bonding. Ordered structures on various length scales endow the hydrogels with additional functions with promising applications in materials science, such as in tissue engineering and mechano-optical sensors. In this review, we briefly describe the recent developments in designing hydrogels with ordered structures of molecular assemblies and the resultant functionalities.

$\mathrm{B}$ iomimetic and bioinspired materials are receiving increasing attention because of their robust functionality and potential applications as artificial tissue $[1,2]$. For natural materials, a high degree of sophistication is achieved through self-organization of the various components into ordered and hierarchical structures following a well-defined pattern [2-4]. Biological tissue possesses these ordered structures, endowing the living organisms with mechanical toughness and functionality, as exemplified by tissues such as bone and cartilage. Therefore, efforts should be made to introduce ordered and hierarchical structure into soft, and also hard, synthetic materials. Among the strategies developed to date, self-assembly is a convenient and powerful method for producing ordered structures [5].

Self-assembly, the spontaneous organization of discrete components into organized structures, is widespread in nature, such as in protein folding and hybridization of the DNA double helix [3-6]. Self-assembled structures usually incorporate additional functionalities. For example, phospholipids form a lipid bilayer around the cell as a barrier to the diffusion of ions and proteins, and tropocollagens self-assemble into fibrils collagens that are closely packed in parallel arrays in the tendon, endowing it with excellent mechanical properties [3]. Inspired by nature, molecular self-assembly that is directed through weak, noncovalent interactions has received tremendous attention for the development of systems with ordered structures and resultant functions $[1,2,6,7]$. These efforts have resulted in various functional materials based on self-assembled structures including solutions, elastomers, gels and hard materials [1,2,7-9].

Gels as a functional system is an emerging field in themselves, possessing a responsiveness to external stimuli such as stress, light, temperature, $\mathrm{pH}$ and ionic strength [10]. Gels with molecular assemblies can be produced in organic solvent (organogels) or aqueous solvent (hydrogels). In this review, we focus primarily on functional hydrogels with self-assembled structures. Functional organogels produced by molecular self-assembly have been covered in several excellent reviews [11-14].

Hydrogels as a functional material have their own advantages over organogels. The aqueous medium makes hydrogels an ideal candidate for soft biotissue, mimicking its functions for applications such as scaffolds for cell culturing $[15,16]$. However, producing structured hydrogels is not easy because the ordered structure formation and water content (which introduces fluctuation) work in opposite directions. The typical kinds of molecules capable of forming self-assembled structures in aqueous media are amphiphile, block-copolymer and liquid-crystalline (LC) molecules. Under certain conditions, the ordered structures formed by molecular self-assembly can be frozen in physically or chemically crosslinked hydrogels with additional functionalities, such as anisotropic optical and mechanical properties. This review considers the close relationship between molecular self-assembly and the resultant specific functions. We highlight the functional LC hydrogels because they possess excellent mechanical, optical and electrical properties but are rarely reviewed in the literature.

\section{Structured hydrogels with biomacromolecular assemblies}

Biomacromolecules such as DNA, filamentous actin (F-actin) and microtubules with rigid or semi-rigid structures and negative charge are significant in the formation of versatile self-assembled structures via noncovalent interaction. It is well known that many cationic proteins, liposomes and multivalent cations are involved in the self-organization of negatively charged semi-rigid biomacromolecules through processes such as cytoskeleton organization and gene packaging [17-19]. Therefore, biomacromolecules are usually extracted and used to synthesize hydrogels via molecular self-assembly. Natural or modified gelatin, chitosan, cellulose, DNA, F-actin, alginate and other biomacromolecules can be triggered to self-assemble by cooling or the addition of salts, bases, polycations or enzymes to form an extended network as a gel state [20-24]. For example, gelatin aqueous solution transitions to a physical gel via cooling to $-30^{\circ} \mathrm{C}$, in which gelatin molecules form triple helical sequences and aggregates as network junction zones to connect the residual peptide sequences in a random conformation. 


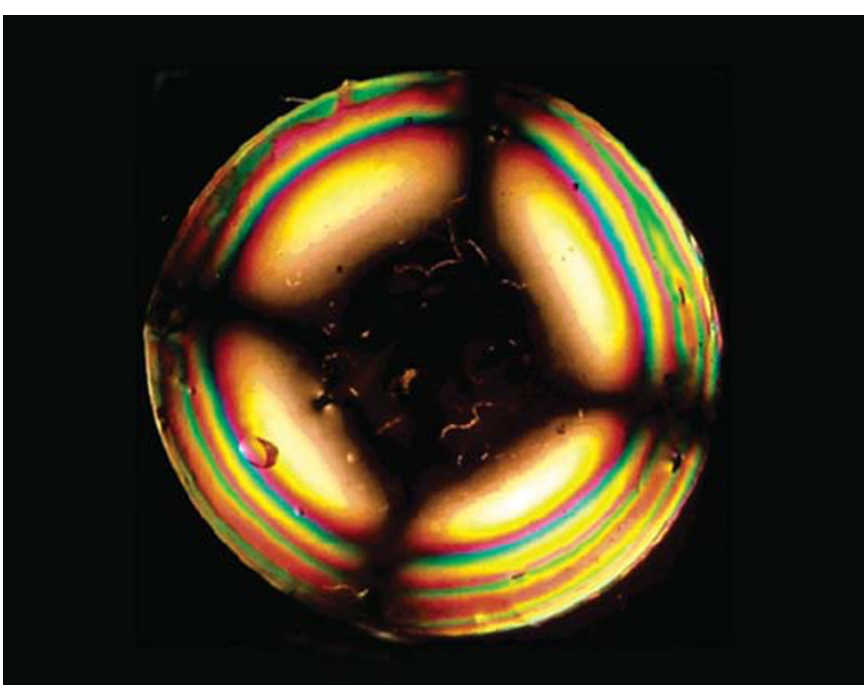

Figure 1. A liquid-crystalline DNA gel observed perpendicular to the long axis of a dialysis tube under crossed polarizers. From Ref. 27 (๔ 2007 ACS).

These biopolymer hydrogels usually have good biocompatibility and biodegradability, and can be used as scaffolds for cell culturing and drug delivery. Regenerated cellulose hydrogel membranes are also strong enough to be used for dialysis in water.

Dobashi and coworkers [25-28] successfully developed a facile dialysis method for fabricating physical biopolymer hydrogels with macroscopic ordered structures. They immersed an aqueous solution of DNA or curdlan inside a semipermeable tube in a multivalent cation solution, such as $\mathrm{Ca}^{2+}$ and $\mathrm{Co}^{2+}$. Biomacromolecules with a semi-rigid structure and negative charge then formed by complexation with the multivalent cations. The cationic diffusion induces and controls the molecular orientation and physical gelation to form radial structures up to the size of several centimeters (Figure 1). These biopolymer molecular self-assembly and gelation systems formed by a dialysis process generally belong to the class of diffusion-limited reaction systems [29]. The macroscopically ordered gels, despite being slightly turbid, show strong birefringence under polarizing microscopy and could be used in optical devices.

In addition to using natural biopolymers to produce functional hydrogels via molecular self-assembly, biopolymers are often modified to precisely tailor the functional group and intermolecular interaction toward a specific chemical reaction for controllable molecular selfassembly, such as by adding a vinyl group or hydrophilic tail [30-32]. Kopecek et al. [31] assembled a hybrid hydrogel from synthetic polymers to produce a structure with a protein-folding motif (coiled coil) that displays temperature-controlled volume shrinkage (Figure 2). They used a linear hydrophilic copolymer as the primary chains with radical polymerization of $N$-(2-hydroxypropyl)-methacrylamide (HPMA) and a metal-chelating monomer $N$-( $N^{\prime}, N^{\prime}$-dicarboxymethylaminopropyl) methacrylamide, where the iminodiacetate- $\mathrm{Ni}^{2+}$ and the terminal histidine residues of the coiled coils anchored as pendant metal-chelating ligands to form metal complexes, as shown in Figure 2(a). Hybrid hydrogels were self-assembled by mixing the coiled-coil proteins and the chelating copolymer of HPMA charged with $\mathrm{Ni}^{2+}$. The gel exhibits temperature-dependent volume change: upon increasing the temperature, the gel suddenly collapses to $10 \%$ of its equilibrium volume at $25^{\circ} \mathrm{C}$ with a transition temperature of $-39^{\circ} \mathrm{C}$, consistent with the melting temperature of the main coiled-coil region (Figure 2(b)). As the temperature increases and the protein unfolds (thermal unfolding), the hydrodynamic radius of the individual pendant protein should decrease from the initial elongated rods of coiled coils to random coils, leading to the collapse of the gel. A hybrid gel with temperature-tunable local junctions formed by molecular self-assembly could be used as a temperature sensor or for therapeutic protein delivery. a

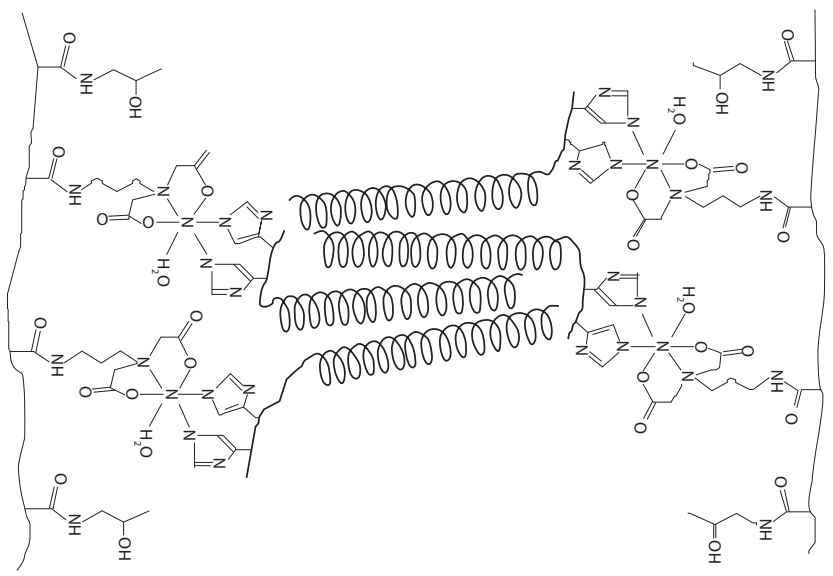

b

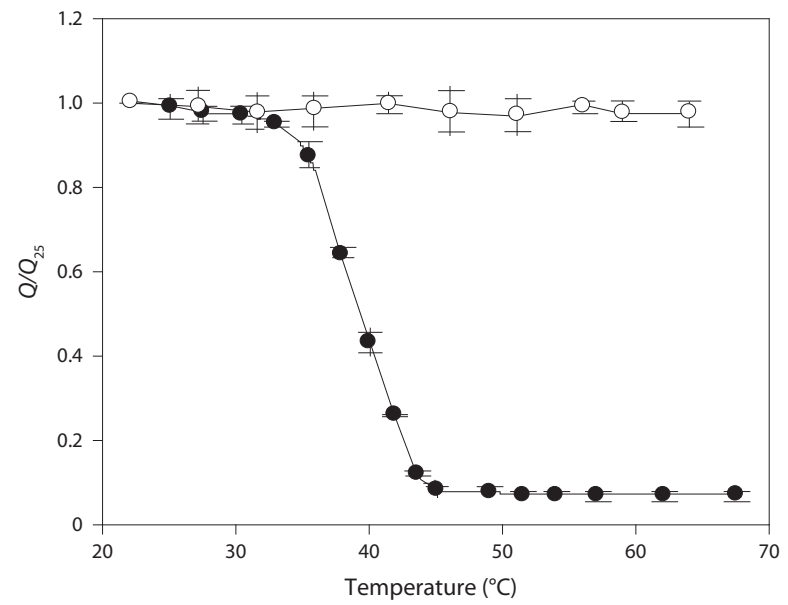

Figure 2. A stimuli-sensitive hybrid hydrogel assembled from synthetic polymers and coiled-coil protein domains. (a) Structural representation of the hybrid hydrogel primary chains and the attachment of terminal histidine residue-tagged coiled-coil proteins. (b) Effect of temperature on gel swelling behavior. $Q / Q_{25}$ is the ratio of the equilibrium volume swelling ratio of gels at an elevated temperature to that at $25^{\circ} \mathrm{C}$ in the same buffer. The filled and open circles denote data for hybrid hydrogels containing proteins with long or short coiled-coil structures, respectively. Adapted from Ref. 31 (๔ 1999 NPG).

\section{Hydrogels with ordered structures produced by self- assembly of synthetic molecules}

Inspired by natural biopolymers that self-assemble to form functional hydrogels, various synthetic molecules with preprogrammed groups and intermolecular interactions have been designed in order to direct the molecular self-assembly to form ordered hydrogel structures with additional functionalities. The molecules that can form self-assembled structures in aqueous media are of three general types: amphiphiles, block copolymers and LC molecules [33]. The self-assembly of amphiphiles and block copolymers generally leads to physical hydrogels that are responsive to environmental change and broadly applicable in drug delivery and cell culturing. Liquid-crystal molecules are usually incorporated into hydrogels by copolymerization or as a dopant in polymerization. These gels show good optical and mechanical properties and are ideal soft actuators.

\section{Self-assembled supramolecular hydrogels}

The physical gelation that forms supramolecular gels is a balance between crystallization and solubilization, and thus the gelator molecule requires functionality that provides both in order to gel a given solvent. 

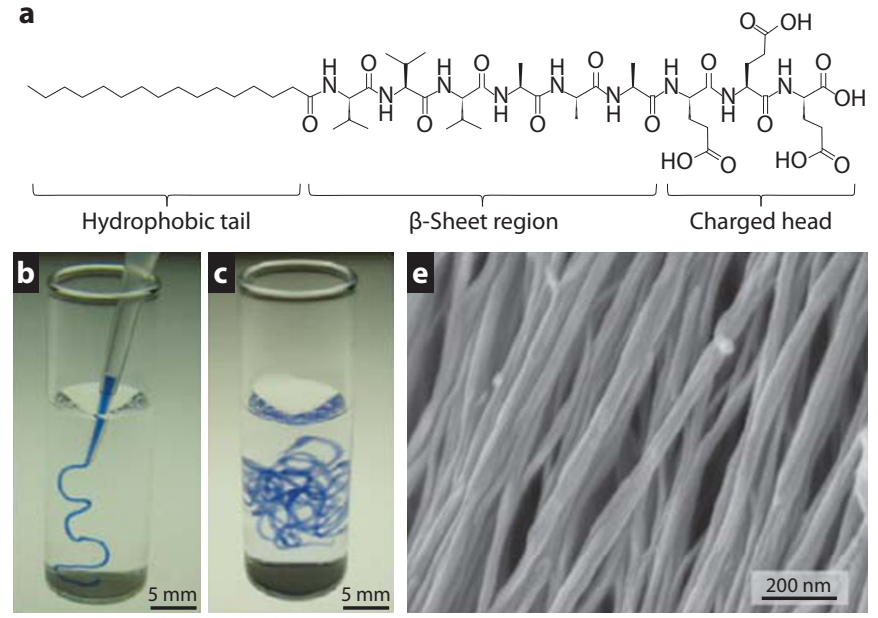

$\beta$-Sheet region
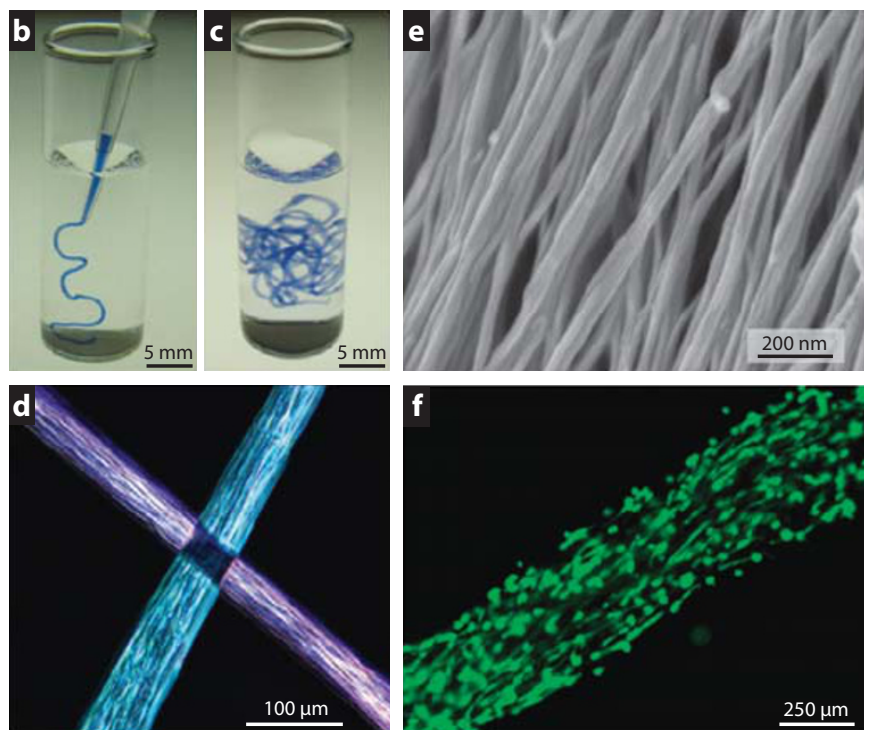

Figure 3. Monodomain gels with long aligned filaments of bundled nanofibers via the self-assembly of peptide amphiphiles. (a) Chemical structure of the peptide amphiphile used. $(b, c)$ A peptide amphiphile solution colored with trypan blue injected into phosphate-buffered saline after heat treatment. (d) Optical polarizing micrograph of two stripe hydrogels. (e) Scanning electron micrograph of aligned nanofiber bundles in macroscopic strings. (f) Fluorescence micrograph of aligned calcein-labeled cells cultured on string. Adapted from Ref. 45 (๔ 2010 NPG).

Therefore, amphiphilic molecules with hydrophobic groups to promote aggregation and hydrophilic groups to support solubility seem to be competent hydrogelators $[34,35]$. There has been much excellent work on the design of specific amphiphiles to form functional hydrogels, in which molecular self-assembly and gelation (formation of branched or entangled fibril networks) are triggered by temperature, $\mathrm{pH}$, ionic strength or an additive such as enzymes or multivalent cations [34-37]. For example, Kimizuka et al. [36] designed cationic L-glutamate derivates as self-assembling receptors that bind anionic fluorescent dyes by moderate electrostatic and van der Waals interactions. Hydrogels were spontaneously formed after mixing the two components. The fluorescent molecules accumulated on the fibrous nanoassemblies, resulting in gels with light-harvesting supramolecular networks. Xu and coworkers [38,39] developed several kinds of supramolecular hydrogels by enzyme-triggered molecular self-assembly. Phosphatase and $\beta$-lactamase were used to control the dephosphorylation and hydrolysis and thus the molecular self-assembly, forming nanofibers and supramolecular hydrogels. These gels show good capacity for the delivery of drugs, detecting the activity of enzymes and controlling the fate of cells.

We introduce here one particular type of amphiphiles, artificial proteins and carbohydrates, and the resultant supramolecular hydrogels that have been studied intensively. Several research groups are active in designing peptides of charged groups of various lengths as hydrophilic heads and hydrophobic tails [40-44]. After specific stimuli triggers, peptides self-assemble to form a secondary structural motif, such as an $\alpha$-helix, $\beta$-hairpin or even extended fibrils of hydrogels. Pochan et al. [41] designed de novo a peptide with a 20 amino acid-long sequence (without a hydrophobic tail) that folded into a $\beta$-hairpin upon heating. These $\beta$-hairpin peptides self-assembled further leading to fibril formation and ultimately hydrogelation. Zhang and coworkers [44] developed a class of amphiphilic peptide hydrogels with high water content that can be used to control the release of functional proteins and the differentiation of cultured cells. Recently, Stupp et al. [45] reported aligned monodomain hydrogels by the molecular self-assembly of peptide-based small molecules on cooling. The molecules in water formed lamellar plaques with a filamentous texture after heat treatment for the spontaneous templated long-range alignment of bundled nanofibers (Figure 3). The designed peptide amphiphiles contained an alkyl tail at the peptide's N-terminus (Figure 3(a)), and self-assembled into high-aspect-ratio nanofibers to form gels with the trigger of ions that screen the charged amino-acid residues [46]. Noodle-like strings could be formed by manually drawing the peptide amphiphile solution after heat treatment $\left(80^{\circ} \mathrm{C}\right.$ for $30 \mathrm{~min}$ then cooling to room temperature) into a salty medium (Figures $3(\mathrm{~b}, \mathrm{c})$ ). Strong birefringence was observed along the strings, suggesting the macroscopic alignment of molecules (Figure 3(d)). The oriented structures were confirmed by scanning electron microscopy (Figure 3(e)), which revealed that the strings contained extraordinarily long arrays of aligned nanofiber bundles. The unheated peptide amphiphile solutions, on the other hand, formed matrices of randomly entangled nanofibers. These strings of aligned nanofibers were used to direct the orientation of cells in threedimensional environments, in which human mesenchymal stem cells (hMSCs) were dispersed in preheated solutions (Figure 3(f)). Both the cell bodies and filopodia were aligned with the nanofiber bundles in the extracellular space. The effects on hMSC orientation possibly resulted from the contact guidance along the preferentially oriented matrix. This work on cellular wires tailed by peptide supramolecular hydrogels shows strong potential for application in regenerative medicine.

Similar to peptide amphiphiles, artificial carbohydrates are also a promising class of hydrogelators. Hamachi and coworkers [47-49] designed a low-molecular-weight gelator containing a glycosylated amino-acid scaffold that directed ordered noncovalent interactions. Thin fibers including extensive hydrophobic domains in their core and an oriented saccharide interface formed via molecular self-assembly and entangled to immobilize water. These gels have been applied in molecular recognition and release, such as for the detection of enzyme activity using semi-wet peptide/protein gel arrays.

\section{Block-copolymer structured hydrogels}

Block copolymers have been widely investigated with the use of aqueous media to form versatile molecular assemblies, including lamellar, micellar, hexagonal cylindrical and bicontinuous gyroid structures, through the different hydrophilicities of the constituent polymers [50]. Under specific conditions, the molecular self-assembly of block copolymers results in extended entangled bundles or condensed junctions that fix the solvent water. Some aqueous solutions of amphiphilic block copolymers exhibit a reversible sol-gel transition if the concentrations are higher than a critical value. These hydrogels usually respond reversibly to changes in temperature, $\mathrm{pH}$ or ionic strength, and are thus useful vehicles for drug delivery and as scaffolds for cell culturing [33,51-56]. For example, the triblock copolymer poly(ethylene oxide)-poly(propylene oxide)-poly(ethylene oxide) (PEO-PPO-PEO; Pluronic) self-assembles into a physical hydrogel with a packed micellar structure at temperatures above $20^{\circ} \mathrm{C}$ [52]. Lemmers and coworkers [56] reported multiresponsive hydrogels based on the charge-driven co-assembly of a triblock copolymer of PEO and poly(3-sulfopropylmethacrylate potassium salt (PSMA), $\mathrm{PSPMA}_{28}-\mathrm{PEO}_{230}-\mathrm{PSPMA}_{28}$, having two charged end blocks with an oppositely charged homopolymer of poly(allylamine hydrochloride) ${ }_{160}$. When the polymer concentrations are above a critical gel concentration, the triblock copolymer will bridge between two different connected micellar cores, leading to the formation of gels. The reversible gels respond to changes in temperature, ionic strength and $\mathrm{pH}$. Kaneko et al. [57] fabricated a thin hydrogel film by molecular self-assembly of a triblock copolymer of long hydrophilic PEO blocks and poly ( $\gamma$-benzyl L-aspartate) (PBLA). The PBLA-b-PEO$b$-PBLA triblock copolymer film cast from methylene chloride was then 
immersed in pure water to form hydrogel membranes with a thickness of $100-200 \mu \mathrm{m}$. These hydrogels contained millimeter-scale spherulitic crystalline domains due to segregation of the hydrophobic PBLA and $\alpha$-helix- $\beta$-strand transformation. The giant crystalline domains in transparent hydrogel membranes endowed the gel with mechanical toughness and good optical properties.

Lamellar structure formed by block copolymers can also be used to fabricate photonic hydrogels after being frozen by chemical crosslinking. Thomas et al. [58,59] developed a photonic hydrogel with broad tunable wavelength range using polystyrene (PS) and quaternized poly(2-vinyl pyridine) (QP2VP) (Figure 4(a)). The block copolymer formed a lamellar structure, and the P2VP blocks were subsequently quaternized and crosslinked to form PS- $b$-QP2VP. The synthesized gel possessed a well-ordered structure with parallel layers, a microdomain periodicity of $100 \mathrm{~nm}$ and approximately equal layer spacing (Figure 4(b)). After swelling the synthesized gels in an aqueous fluid reservoir, the glassy hydrophobic layer of PS prohibited swelling in the direction parallel to the lamellar layers, thus forcing expansion of the hydrophilic layer of P2VP along the layer normal, leading to extremely large optical tunability through changes in both layer spacing and refractive index. These one-dimensional Bragg stacks reflect incident light from the ultravioletvisible region to the near-infrared $(350-1,600 \mathrm{~nm})$. Multicolor patterns were formed by the sequential coating and crosslinking of the gel films (Figure 4(c)). These photonic gels show promise for applications involving colorimetric sensors, tunable optically pumped lasers, photonic switches and multiband filters.

We mention here another kind of copolymer hydrogel, although it does not belong to the class of block copolymers. Long alkyl side chains are anchored on the primary chains by the copolymerization of alkyl acrylates and another hydrophilic monomer. In early studies, hydrogels with crystalline domains were synthesized by copolymerizing acrylic acid (AA) and stearyl acrylate (SA) or acryloylhexadecanoic acid (AHA) $[60,61]$. The poly(SA-co-AA) and poly(AHA-co-AA) hydrogels show strong birefringence because of the aggregation and crystallization of side chains. Interestingly, the poly(SA-co-AA) hydrogel has shape memory due to the temperature-driven order-disorder transitions of SA-rich domains in water. Firestone and coworkers [62] designed and polymerized a methylimidazolium-based ionic liquid that incorporated an acryloyl moiety at the terminus of a $\mathrm{C}_{8}$ alkyl chain. The ionic liquid monomer weakly self-assembled to form a lamellar structure upon addition of water and oligomerized on mild heating to be frozen by the subsequent chemically crosslinking reaction. The lamellar spacing could be tuned by adjusting the water content of the gels.

\section{Liquid-crystalline hydrogels}

The examples mentioned above are almost all of physical hydrogels (except the photonic block copolymer gel), in which gelation occurs simultaneously with the molecular self-assembly process by creating extended, branched or entangled networks to immobile water molecules. These gels are responsive to external stimuli (e.g. temperature, ionic strength, and $\mathrm{pH}$ ) and good for cell culture and drug delivery. However, their poor mechanical toughness limits their applications in other situations such as soft robotics (chemomechanical systems). Liquid-crystalline molecules and their assemblies have excellent optical, electric and magnetic properties and can be incorporated in physical and chemical hydrogels as a monomer or dopant during the polymerization. These gels have relatively good mechanical properties and are widely used in developing chemomechanical actuators, such as mechano-optical sensors. Several examples are given below to illustrate the promising prospects of LC hydrogels as functional materials.

Liquid-crystalline monomers can be polymerized individually or copolymerized with other monomers to form chemically or physically crosslinked hydrogels (with or without crosslinker), in which mesogens can be nested on the primary polymer chains or anchored on primary chains as pendants. In our previous work, water-swollen LC hydrogels were synthesized by copolymerization of AA and an LC monomer
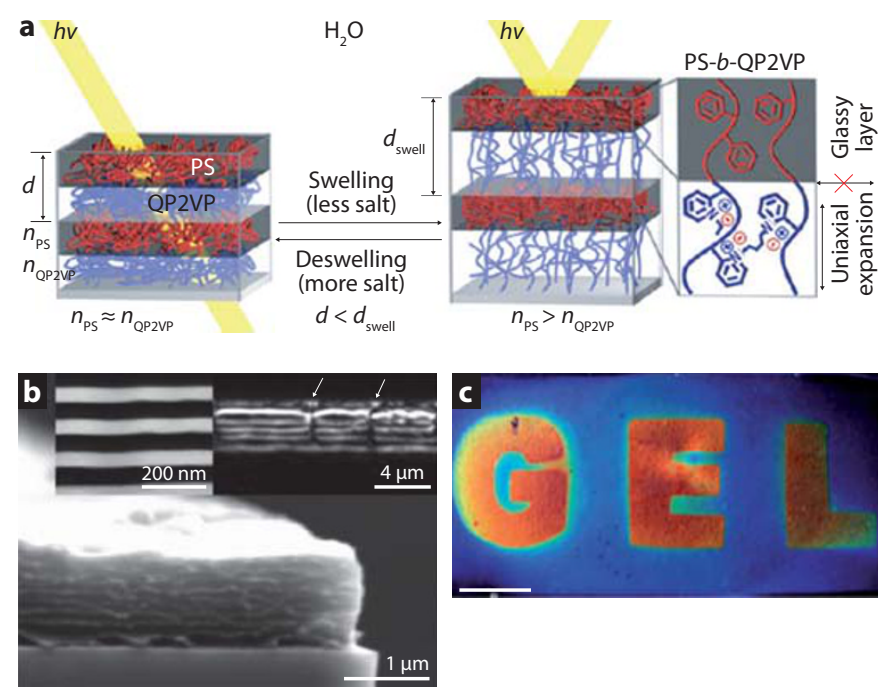

Figure 4. (a) Schematic diagram of the structure of a photonic gel film and the tuning mechanism. The photonic gel film was prepared by self-assembly of a diblock copolymer (PS-b-QP2VP). The QP2VP gel layers swell or deswell (blue) in response to changes in aqueous solvent to tune the domain spacing, the refractive-index contrast and thus the wavelengths of light reflected by the stop band. (b) Scanning electron micrograph of a dry PS-b-QP2VP lamellar photonic film deposited on a silicon wafer. (c) Photograph of the photonic gel film immersed in water. Adapted from Ref. 58 (๔ 2007 NPG).

11-(4'-cyanobiphenyloxy)undecyl acrylate (11CBA) in dimethylformamide solution [63-66]. The states of poly(11CBA-co-AA) after being swollen in water depended on the monomer compositions and water content, displaying multiphase behavior (Figure 5(a)) [64]. At room temperature, the copolymers dissolved in water when the molar fraction of $11 \mathrm{CBA}\left(F_{11 \text { Св }}\right)$ was below 0.18 . At $F_{11 \text { Св }}>0.26$, the copolymers swelled to form hydrogels due to the hydrophobic interaction between mesogenic side chains, resulting in the formation of a physical crosslinking junction. The hydrogels showed a smectic $\mathrm{A}(\mathrm{SmA})$ structure at low water content $\left(w_{\mathrm{H}_{2} \mathrm{O}}\right)$ and $F_{11 \text { CBA }}>0.26$, in which the side chains formed bilayers aligned perpendicularly to the main-chain axis. However, at $F_{11 \mathrm{CBA}}=0.26$ and $w_{\mathrm{H}_{2} \mathrm{O}}=70 \mathrm{wt} \%$, the SmA structure was transformed into an amorphous state. The gels exhibited a smetic I (SmI) structure, with side chains tilted to the normal direction of the main chains, in a narrow range where $F_{11 \text { СBA }}>0.29$ and $w_{\mathrm{H}_{2} \mathrm{O}}>13 \mathrm{wt} \%$. Furthermore, the SmI structure of the hydrogels (at $F_{11 \text { СвA }}=0.37$ ) could be transitioned backward to the $\mathrm{SmA}$ phase by mechanical elongation at $25^{\circ} \mathrm{C}$ accompanied by necking $[65,66]$. This $\mathrm{SmI}$ gel structure induced by mechanical stretching was not stable at high temperature. Upon heating, the stretched gel fiber changed its structure from SmI to SmA with a transition temperature of $40^{\circ} \mathrm{C}$, corresponding to the smectic mesophase transition of the poly(11CBA-co-AA) (Figure 5(b)). Simultaneously, the stretched hydrogel fiber dramatically shrank during this transition, possibly because it increased the molecular mobility in the hydrophobic junctions and relaxed the residual internal stress (Figure 5(c)). Structured gels that show anisotropic shrinkage in one direction with increasing temperature are promising for use as soft actuators.

In addition to the physical LC hydrogels, LC molecules can be introduced into chemical hydrogels by copolymerization with other monomers or as a dopant. In recent years, we have focused on the synthesis of LC hydrogels using a semi-rigid polyanion poly(2,2'-disulfonyl-4,4'-benzidine terephthalamide) (PBDT) as a dopant [67-75]. PBDT has high molecular weight, negative charge and a semi-rigid structure, similar to natural biopolymers, and aqueous solutions of PBDT show a nematic LC state above a significantly low critical concentration $\left(C_{\mathrm{LC}}^{*}\right)$ of $2-2.8 \mathrm{wt} \%$. We found that the viscosity of PBDT solutions increases dramatically when the concentrations are above the overlap 


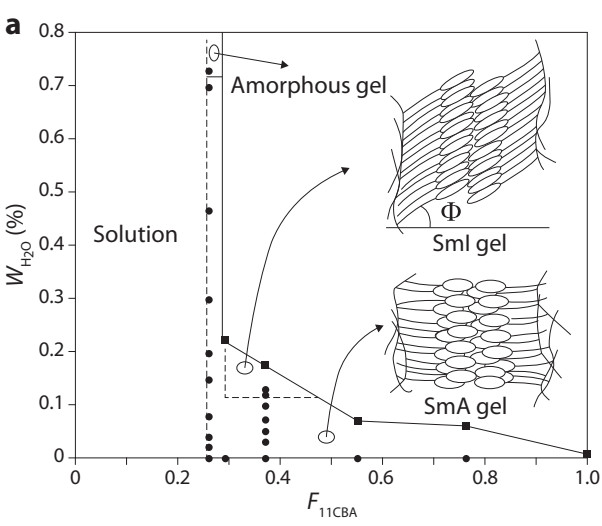

b

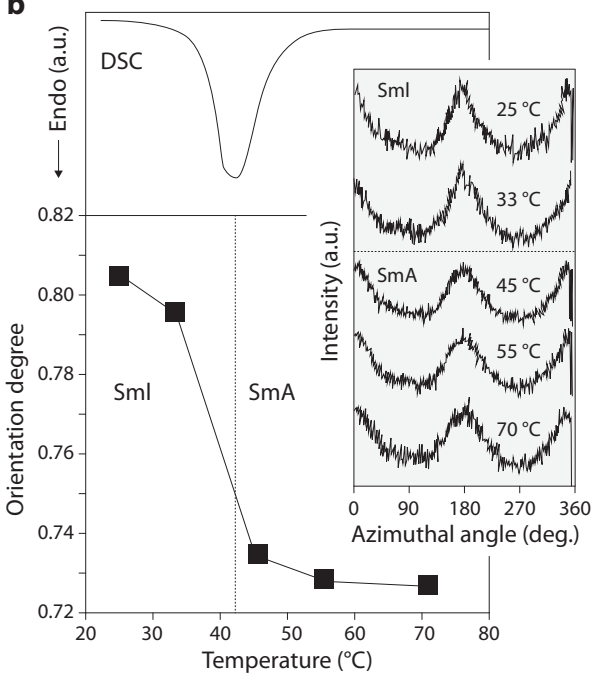

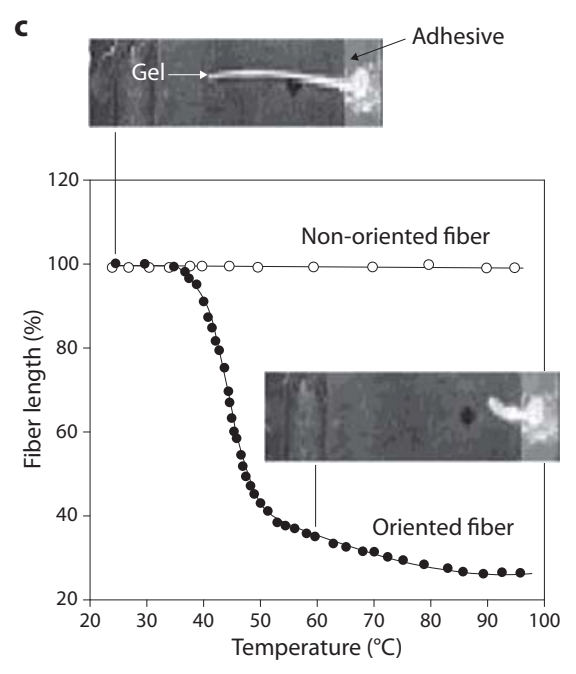

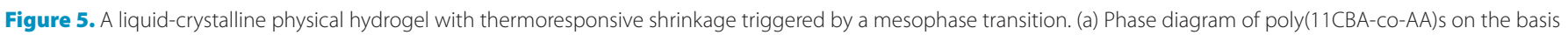

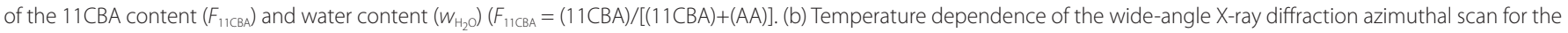

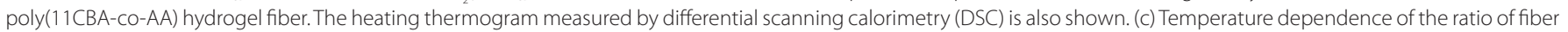
length to the original length of the poly(11CBA-CO-AA) hydrogel fiber, oriented and unoriented. Adapted from Refs 64 (৫ 2000 ACS) and 66 (৫ 2004 ACS).

concentration of $0.2-0.3 \mathrm{wt} \%$ due to chain entanglement $[67,70]$. PBDT is an optically positive liquid crystal; it shows blue and orange colors under crossed polarizers when oriented in the southwest and northwest directions, respectively. If we polymerize a cationic monomer and chemical crosslinker in the presence of PBDT as a dopant, the synthesized polycations will interact with the oppositely charged PBDT to form a semi-rigid polyion complex and self-assemble into ordered structures that are frozen in the hydrogels. The two structured PBDTcontaining hydrogels mentioned below are based on the formation and self-assembly of such a semi-rigid polyion complex.

The chemical structures of PBDT and the cationic monomer $\mathrm{N}$-[3( $N, N$-dimethylamino)propyl] acrylamide methyl chloride quaternary (DMAPAA-Q) are shown in Figure 6(a) [68]. The PBDT used here has a molecular weight of $1.8 \times 10^{6} \mathrm{~g} \mathrm{~mol}^{-1}$ and $C_{\mathrm{LC}}^{*}$ of $2.2 \mathrm{wt} \%$. DMAPAA-Q will form a flexible polycation after polymerization. The hydrogels were synthesized by photo-initiated polymerization at room temperature with different monomer concentrations $\left(C_{\mathrm{Q}}\right)$ and PBDT concentrations $\left(C_{\mathrm{P}}\right)$ while keeping the chemical crosslinker concentration at $2 \mathrm{~mol} \%$ and photoinitiator concentration at $0.15 \mathrm{~mol} \%$ (both relative to the monomer). The precursor aqueous solutions were transparent and optically isotropic at $C_{\mathrm{P}}<C_{\mathrm{LC}}$. However, the hydrogels synthesized with relatively low $C_{\mathrm{Q}}\left(C_{\mathrm{Q}}<1.5 \mathrm{M}\right)$ were turbid, becoming transparent at relatively high $C_{\mathrm{Q}}\left(C_{\mathrm{Q}} \geq 2 \mathrm{M}\right)$ (Figure $6(\mathrm{~b})$ ). The transparent gels exhibited strong birefringence $\left(\Delta n>10^{-5}\right)$ with anisotropic domains of $1-3 \mathrm{~mm}$ in size at $C_{\mathrm{Q}}>1.75 \mathrm{M}$ and $C_{\mathrm{P}}>0.5 \mathrm{wt} \%$ (Figure 6(d)). On the other hand, the turbid gels, indicating the occurrence of phase separation, possessed a unique well-ordered network-like structure of micrometer size, despite exhibiting weak birefringence (Figure 6(c)). Both the anisotropic and network-like structures could be maintained after swelling the gels in pure water.

We elucidate the structure formation mechanism of gels as follows $[68,69]$. When polymerization starts, the generated polycation interacts with the semi-rigid anionic PBDT to form a polyion complex that self-assembles into small anisotropic aggregates with high viscosity. When the reacted solution has low ionic strength $\left(C_{\mathrm{Q}} \leq 1.5 \mathrm{M}\right)$, the polyion complexes are unstable and phase separation occurs. The phase separation rapidly develops into viscoelastic phase separation owing to the dynamical asymmetry, forming a network-like structure of $-40 \mu \mathrm{m}$ in size, which is permanently frozen by the subsequent chemical crosslinking reaction. On the other hand, if the solution has high ionic strength $\left(C_{\mathrm{Q}} \geq 2 \mathrm{M}\right)$, no severe phase separation will occur. The small polyion
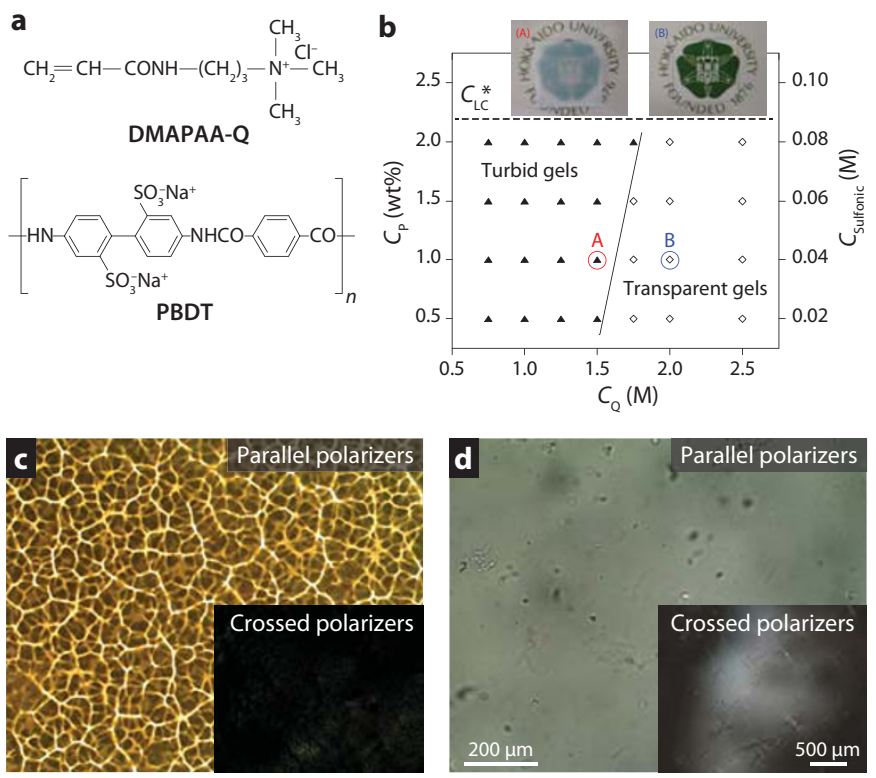

Figure 6. Structured hydrogels synthesized by polymerization of a cationic monomer in the presence of a small amount of semi-rigid polyanion as a dopant. (a) Chemical structures of DMAPAA-Q and PBDT. (b) Appearance (inset) of as-prepared gels QP- $C_{Q^{-}}$ $C_{P}-0.15$ synthesized with various concentrations $C_{Q}$ and $C_{P}\left(C_{\text {sulfonic, }}\right.$ concentration of sulfonic moeity in PBDT). (c,d) Optical micrographs of as-prepared gels under parallel and crossed polarizers: (c) $C_{p}=1, C_{Q}=1.5$, (d) $C_{p}=1, C_{Q}=2$. Adapted from Ref. 72 (c) 2009 Wiley-VCH).

complex aggregates self-assemble further to form macroscopically anisotropic domains of $1-3 \mathrm{~mm}$ in size that are frozen by subsequent gelation to form anisotropic hydrogels. These hydrogels with a micrometer-scale network-like structure and millimeter-scale anisotropic domains are novel and are expected to play a crucial role in materials science, such as in bionics and optical devices.

As suggested by the findings above regarding PBDT-containing hydrogels with millimeter-scale anisotropic domains, if we could control the self-assembly direction of the polyion complex, it would be possible to develop a hydrogel with a macroscopically ordered structure. A strategy we have taken [74] is to polymerize the precursor solution 
a

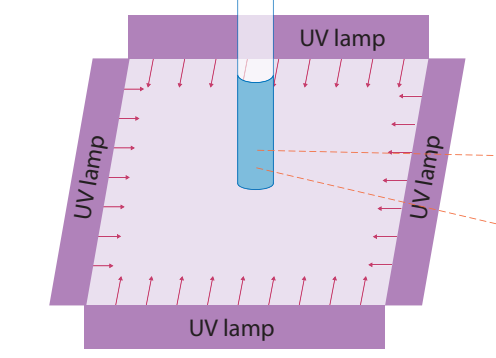

b
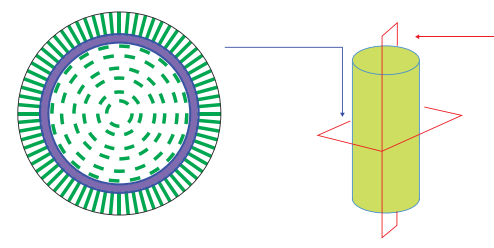
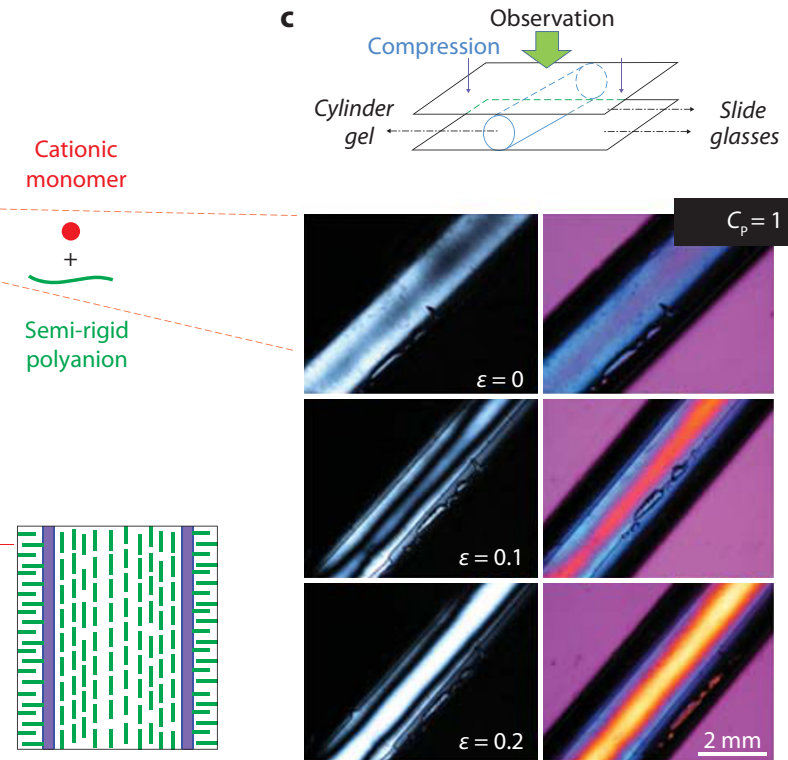

monomer

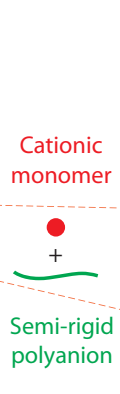

polyanion

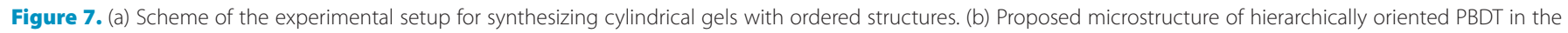

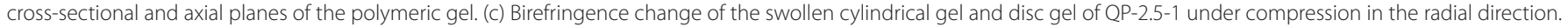
The right columns show observations with the insertion of a $530 \mathrm{~nm}$ sensitive tint plate. The strain ( $\varepsilon$ ) is noted in each panel. Adapted from Ref. 74 (๔ 2009 ACS).

by ultraviolet irradiation from all sides, as shown in Figure 7(a). The reaction should proceed from the outer region to the central region that controls the self-assembly direction, and could result in macroscopically structured hydrogels. In the experiments, the precursor solutions were poured into a glass tube placed vertically in the center of a square arrangement of four ultraviolet lamps. After polymerization, the as-prepared gel was transparent and swelled in $1 \mathrm{M}$ sodium chloride solution (used to prevent over-swelling). The precursor solution showed no birefringence. However, the as-prepared gel exhibited strong birefringence that was maintained upon swelling. A thin disc of the gel cut from the bulk cylinder showed a huge Maltese cross that did not change while rotating the sample, indicating that PBDT molecules had formed a cylindrical symmetric structure during polymerization. We further characterized the microstructure of PBDT and its polyion complex in the cylindrical hydrogels by polarizing microscopy with a $530 \mathrm{~nm}$ tint plate, and by small-angle X-ray scattering. The proposed structure model is shown in Figure 7(b). PBDT and its polyion complex self-assembled into a radial structure in the outer region of the cylinder gels, whereas in the inner region, they formed a concentric structure and aligned parallel with the axial direction. We demonstrated that the radial (homeotropic) orientation of the semi-rigid polyion complex in the outer region of the gel was due to interfacial interaction with the glass wall. In the inner region, the complex was oriented in concentric and axial directions due to heterogeneous polymerization (through light absorbance by the PBDT), which led to monomer diffusion and orientation of the polyion complexes perpendicular to the diffusion direction.

These gels with complex ordered structures exhibit a sensitive response to external forces. We observed the birefringence change of the cylinder and disc gels under compression in the radial direction, as shown in Figure $7(\mathrm{c})$. The swollen cylindrical gel $\left(C_{\mathrm{Q}}=2.5 \mathrm{M}, C_{\mathrm{P}}=1 \mathrm{wt} \%\right)$ showed strong birefringence before compression, and the birefringence color with the tint plate inserted indicated that the axial alignment of PBDT predominated in observations from the radial direction. With applied compression, the birefringence color of the central region changed from blue to orange, indicating that the preferential axial alignment was overwhelmed by that in the concentric direction. This is attributed to compressional enhancement of the concentric directional alignment of PBDT due to lateral stretch and a shortening of axial length as a result of a reduction in thickness. Furthermore, the birefringence of the disc gels was observed to change under compression from both sides.
Micrographs of the disc gels showed a circular symmetric Maltese cross and a black ring before compression. With applied compression force, the intensity and range of blue birefringence in the inner region gradually decreased, and those of the orange birefringence increased. The compression promoted the alignment of PBDT perpendicular to the force direction due to lateral stretching, causing the concentric alignment in the inner region of the disc gel to gradually change from circular to elliptic. Structured gels showing sensitive response to external force through changes in birefringence color are expected to find applications in mechano-optical sensors and open avenues in the development of biomimetic hydrogels with intricate, complex structures.

Another PBDT-containing hydrogel with macroscopic anisotropic structure and excellent extensibility was developed by a two-step approach [71]. A physical anisotropic hydrogel was synthesized by controlling the diffusion of $\mathrm{Ca}^{2+}$ in PBDT aqueous solutions. The physical gel was then immersed in an acrylamide (AAm) precursor solution and polymerized to form a second flexible network with high entanglement. The double-network hydrogels possess both optical anisotropy and mechanical toughness.

In the LC hydrogels described above, ordered molecular assemblies were formed during polymerization, in which the coupling between molecular self-assembly and gelation was important for producing the final ordered structure and resultant functions. Additionally, lyotropic LC molecules with oriented assemblies such as lamellar and hexagonal columns can be employed as templates to fabricate structured hydrogels $[76,77]$. After removing the template, such hydrogels possess an ordered porous structure and good permeability, and therefore act as good scaffolds for cell culturing.

Recently, we reported a multifunctional anisotropic hydrogel containing a membrane-like lamellar bilayer structure with unidirectional alignment [78]. Lipid-like bilayers of an LC surfactant were immobilized in polyacrylamide (PAAm) hydrogels with high anisotropic swelling ratio and elastic modulus, and mechanically tunable structural color. A system consisting of a polymeric surfactant, dodecylglyceryl itaconate (DGI), and PAAm was initially developed by Tsujii and colleagues $[79,80]$. The DGI monomers self-assembled into periodically stacked bilayers displaying behavior similar to that of lamellar liquid crystal in water or in the precursor solution containing AAm, crosslinker and initiator. After polymerization, the lamellar structure of the polymeric-DGI (PDGI) was immobilized inside the PAAm network 


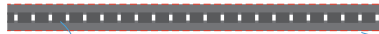

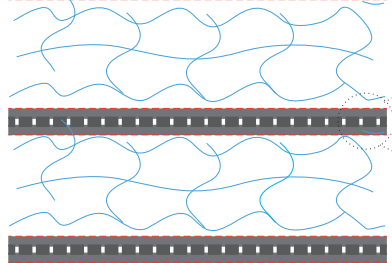

00000000 $\{\{\{\}\{\{\}\}$

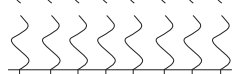
10000000

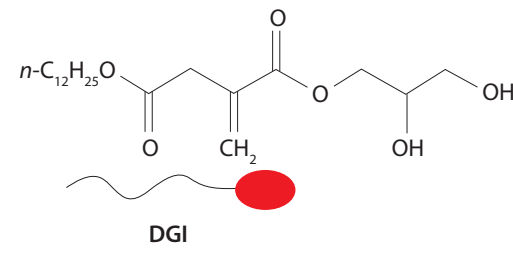
$\mathrm{OH}$

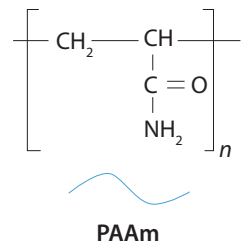

PAAm b

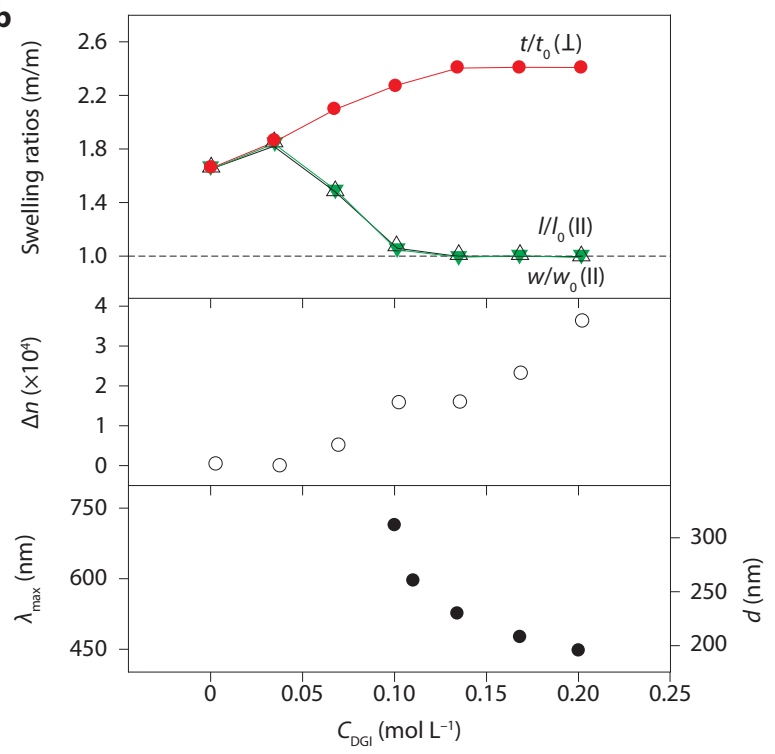

c

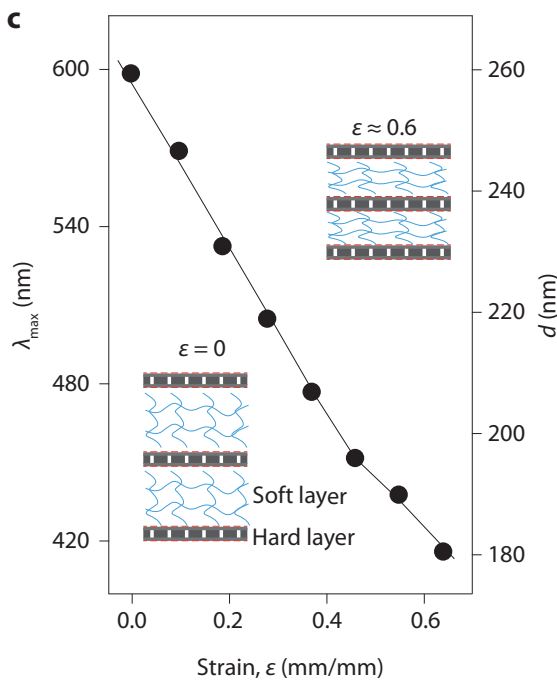

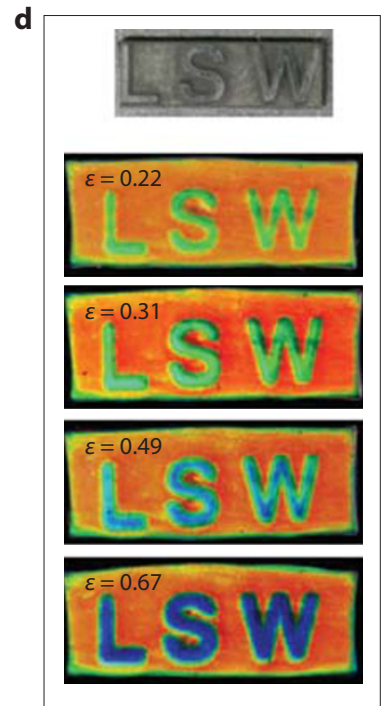

Figure 8. (a) Scheme for the synthesis of a polyacrylamide hydrogel with unidirectional alignment of lamellar bilayers. (b) Change in swelling ratio, birefringence ( $\Delta n$ ), Bragg reflection peak wavelength $\left(\lambda_{\max }\right)$ and lamellar distance $(d)$ at an incident angle of $60^{\circ}$ for gels synthesized with various DGI concentrations. (c) Relationship of $\lambda_{\max }$ and $d$ with compressional strain $(\varepsilon)$ of the gel. The compressional strain is mainly localized in the soft PAAm network layers of the gel, which causes a decrease in lamellar distance in the perpendicular direction. (d) The letters 'LSW' in a rubber stamp (top) cause a color change in a gel sheet when pressed onto the stamp (lower panels). The color change is dependent on the compressional force applied (g). Adapted from Ref. 78 (๑ 2010 Wiley-VCH).

structure. However, the self-assembled lamellar domains were randomly dispersed in the precursor solutions and the synthesized gels were isotropic on a macroscopic level.

We developed a method to successfully form a macroscopic singledomain lamellar structure of DGI by applying a shear flow to the precursor solution between two parallel glass walls prior to polymerization [78]. The as-prepared plate-like bulk hydrogel with a thickness of $-1 \mathrm{~mm}$, containing about 4,000 PDGI bilayers with unidirectional alignment (Figure 8(a)), showed perfect anisotropic swelling behavior: the gel only swelled in the direction perpendicular to the lamellar bilayers. The gel also possessed strong mechanical anisotropy with a Young's modulus in the direction of the lamellar layers ten times higher than that in the perpendicular direction. Moreover, the hydrogel exhibited excellent visible coloration due to Bragg reflection on the multilayer planes of PDGI (Figure 8(b)). The high mechanical strength of the gels permitted their structural color to be reversibly fine-tuned over a large spectral range by controlling the mechanical stress/strain. The color as well as the reflection peak shifted from the red to blue wavelength region with increasing induced strain and hence decreasing lamellar distance. The wavelength maximum decreased from $-600 \mathrm{~nm}$ to $-415 \mathrm{~nm}$ as the strain of the gel increased from 0 to 0.64 (Figure 8 (c)). Since the gel had a structure comprised of two alternating layers with large difference in elastic modulus, almost all the compressive deformation was imposed on the soft PAAm layers, resulting in a decrease in the lamellar distance and no compressive deformation of the hard PDGI lamellar bilayers except lateral expansion.

Due to this widely tunable structural color, these gels could be potentially used as mechano-optical actuators such as stress/strain sensors and deformation-based color displays. For example, when the gel was compressed on a hard, uneven or engraved substrate, the localized gel showed different colors depending on the appearance of the substrate and the induced compressive strain of the gel. As shown in Figure 8(d), this behavior allows the characters 'LSW' to be produced distinctly in the gel in a different color by compressing the gel on a rubber stamp bearing those letters. The compressive strain in this case was exerted only in the engraved regions, and the color in the deformed portion of the gel shifted to blue as the strain increased.

\section{Conclusions}

The development of functional hydrogels with ordered molecular assemblies is an emerging research field. Various novel structured hydrogels with distinct properties have been developed, giving rise to specific applications. Empirically, physical supramolecular hydrogels formed by molecular self-assembly are responsive (undergo a sol-gel transition) to external stimuli such as temperature, $\mathrm{pH}$ and ionic strength, yet are generally mechanically weak. These gels draw attention for applications as vehicles for drug delivery, molecular control and release, and as scaffolds for cell culturing. On the other hand, chemically crosslinked hydrogels embedded with ordered LC assemblies possess particular optical and electric properties. Specially, the relatively good mechanical properties of such LC hydrogels permits their use in chemomechanical systems and soft actuators. As more self-assembling systems are developed toward achieving specific functionalities, we believe that structured hydrogels will attract more intensive attention as functional materials, especially for soft actuators mimicking biotissue and as three-dimensional hierarchical scaffolds for guiding cell culturing and differentiation to form living organisms. 


\section{References}

1. S. Zhang, Nature Biotechnol. 21, 1171 (2003)

2. C. Sanchez, H. Arribart, M. M. G. Guille, Nature Mater. 4, 277 (2005).

3. L. P. Gartner, J. L. Hiatt, Color Textbook of Histology, 2nd ed. (Saunders, USA, 2001).

4. K. McGrath, D. S. Kaplan, Protein-Based Materials (Bikhauser, USA, 1997).

5. G. M. Whitesides, B. Grzybowski, Science 295, 2418 (2002).

G. M. Whitesides, J. P. Mathias, C. T. Seto, Science 254, 1312 (1991).

T. Kato, Science 295, 2414 (2002).

M. A. C. Stuart et al., Nature Mater. 9, 101 (2010).

I. Dierking, Adv. Mater. 12, 167 (2000).

10. Y. Osada, J.-P. Gong, Adv. Mater. 10, 827 (1998).

11. M. George, R. G. Weiss, Acc. Chem. Res. 39, 489 (2006).

12. T. Kato, Y. Hirai, S. Nakaso, M. Moriyama, Chem. Soc. Rev. 36, 1857 (2007).

13. A. Ajayaghosh, V. K. Praveen, Acc. Chem. Res. 40, 644 (2007).

14. M.-O. M. Piepenbrock, G. O. Lloyd, N. Clarke, J. W. Steed, Chem. Rev. 110, 1960 (2010).

15. J. L. Drury, D. J. Mooney, Biomaterials 24, 4337 (2003).

16. M. P. Lutolf, J. A. Hubbell, Nature Biotechnol. 23, 47 (2005).

17. J. O. Rädler, I. Koltover, T. Salditt, C. R. Safinya, Science 275, 810 (1997).

18. L. S. Hirst, C. R. Safinya, Phys. Rev. Lett. 93, 018101 (2004).

19. H. J. Kwon et al., Langmuir 23, 6257 (2007).

20. S. B. Ross-Murphy, Polymer 33, 2622 (1992).

21. A. Aggeli et al., Nature 386, 259 (1997).

22. A. Di Martino, M. Sittinger, M. V. Risbud, Biomaterials 26, 5983 (2005).

23. K. Lee, D. Kaplan, Adv. Biochem. Eng. Biothechnol. 102, 187 (2006).

24. S. H. Um et al., Nature Mater. 5, 797 (2006).

25. T. Dobashi, M. Nobe, H. Yoshihara, T. Yamamoto, A. Konno, Langmuir 20, 6530 (2004)

26. M. Nobe, T. Dobashi, T. Yamamoto, Langmuir 21, 8155 (2005).

27. T. Dobashi, K. Furusawa, E. Kita, Y. Minamisawa, T. Yamamoto, Langmuir 23, 1303 (2007)

28. K. Furusawa, Y. Minamisawa, T. Dobashi, T. Yamamoto, J. Phys. Chem. B 111, 14423 (2007)

29. B. A. Grzybowski, K. J. M. Bishop, C. J. Campbell, M. Fialkowski, S. K. Smoukov, Soft Matter 1, 114 (2005).

30. W. A. Petka, J. L. Harden, K. P. McGrath, D. Wirtz, D. A. Tirrell, Science 281, 389 (1998)

31. C. Wang, R. J. Stewart, J. Kopeček, Nature 397, 417 (1999).

32. A. P. Nowak et al., Nature 417, 424 (2002).

33. H. J. Chung, T. G. Park, Nano Today 4, 429 (2009).

34. L. A. Estroff, A. D. Hamilton, Chem. Rev. 104, 1201 (2004).

35. A. R. Hirst, B. Escuder, J. F. Miravet, D. K. Smith, Angew. Chem. Int. Ed. 47, 8002 (2008)

36. T. Nakashima, N. Kimizuka, Adv. Mater. 14, 1113 (2002).

37. J. W. Steed, Chem. Soc. Rev. 39, 3686 (2010).

38. Z. Yang, G. Liang, B. Xu, Acc. Chem. Res. 41, 315 (2008).

39. Y. Gao, F. Zhao, Q. Wang, Y. Zhang, B. Xu, Chem. Soc. Rev. 39, 3425 (2010).

40. J. D. Hartgerink, E. Beniash, S. I. Stupp, Proc. Natl Acad. Sci. USA 99, $5133(2002)$
41. B. Ozbas, K. Rajagopal, J. P. Schneider, D. J. Pochan, Phys. Rev. Lett. 93, 268106 (2004)

42. T. J. Deming, Soft Matter 1, 28 (2005).

43. R. P. Nagarkar, R. A. Hule, D. J. Pochan, J. P. Schneider, J. Am. Chem. Soc 130, 4466 (2008).

44. S. Koutsopoulos, L. D. Unsworth, Y. Nagai, S. Zhang, Proc. Natl Acad. Sci. USA 106, 4623 (2009).

45. S. Zhang et al., Nature Mater. 9, 594 (2010).

46. R. M. Capito, H. S. Azevedo, Y. S. Velichko, A. Mata, S. I. Stupp, Science 319, 1812 (2008).

47. S. Kiyonaka et al., Nature Mater. 3, 58 (2004).

48. A. Wada, S. Tamura, M. Ikeda, I. Hamachi, J. Am. Chem. Soc. 131, 5321 (2009).

49. H. Komatsu et al., J. Am. Chem. Soc. 131, 5580 (2009).

50. F. S. Bates, G. H. Fredrickson, Annu. Rev. Phys. Chem. 41, 525 (1990).

51. T. Kissel, Y. Li, F. Unger, Adv. Drug. Delivery Rev. 54, 99 (2002).

52. S. Fusco, A. Borzacchiello, P. A. Netti, J. Bioact. Compat. Polym. 21, 149 (2006).

53. J.-H. Ryu, M. Lee, J. Am. Chem. Soc. 127, 14170 (2005)

54. J. Li et al., Biomaterials 27, 4132 (2006).

55. C. He, S. W. Kim, D. S. Lee, J. Control. Rel. 127, 189 (2008).

56. M. Lemmers, J. Sprakel, I. K. Voets, J. van der Gucht, M. A. C. Stuart, Angew. Chem. Int. Ed. 49, 708 (2010).

57. T. Kaneko, S. Tanaka, A. Ogura, M. Akashi, Macromolecules 38, 4861 (2005).

58. Y. Kang, J. J. Walish, T. Gorishnyy, E. L. Thomas, Nature Mater. 6, 957 (2007).

59. C. Kang et al., J. Am. Chem. Soc. 131, 7538 (2009).

60. Y. Osada, A. Matsuda, Nature 376, 219 (2002).

61. M. Uchida, M. Kurosawa, Y. Osada, Macromolecules 28, 4583 (1995).

62. D. Batra, D. N. T. Hay, M. A. Firestone, Chem. Mater. 19, 4423 (2007).

63. T. Kaneko, K. Yamaoka, J. P. Gong, Y. Osada, Macromolecules 33, 412 (2000).

64. T. Kaneko, K. Yamaoka, J. P. Gong, Y. Osada, Macromolecules 33, 4422 (2000).

65. K. Yamaoka, T. Kaneko, J. P. Gong, Y. Osada, Langmuir 19, 8134 (2003).

66. T. Kaneko, K. Yamaoka, Y. Osada, J. P. Gong, Macromolecules 37, 5385 (2004).

67. T. Funaki et al., Langmuir 20, 6518 (2004).

68. Y. Shigekura et al., Adv. Mater. 17, 2695 (2005).

69. Y. Shigekura et al., Macromolecules 40, 2477 (2007).

70. W. Yang et al., Macromolecules 41, 1791 (2008).

71. W. Yang, H. Furukawa, J. P. Gong, Adv. Mater. 20, 4499 (2008).

72. Z. L. Wu, H. Furukawa, W. Yang, J. P. Gong, Adv. Mater. 21, 4696 (2009).

73. Z. L. Wu, T. Kurokawa, S. Liang, J. P. Gong, Macromolecules 43, 8202 (2010).

74. Z. L. Wu, T. Kurokawa, S. Liang, H. Furukawa, J. P. Gong, J. Am. Chem. Soc. 132, 10064 (2010).

75. Z. L. Wu, M. Arifuzzaman, T. Kurokawa, H. Furukawa, J. P. Gong, Soft Matter 7, 1884 (2011)

76. M. Antonietti, C. Göltner, H.-P. Hentze, Langmuir 14, 2670 (1998).

77. J. D. Clapper, C. A. Guymon, Adv. Mater. 18, 1575 (2006).

78. M. A. Haque, G. Kamita, T. Kurokawa, K. Tsujii, J. P. Gong, Adv. Mater 22, $5110(2010)$

79. K. Naitoh, Y. Ishii, K. Tsujii, J. Phys. Chem. 95, 7915 (1991).

80. K. Tsujii, M. Hayakawa, T. Onda, T. Tanaka, Macromolecules 30, 7397 (1997).

\section{Author profiles}

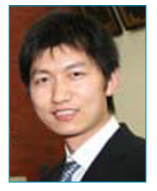

\section{Zi Liang Wu}

Zi Liang Wu graduated from Zhejiang University in China in 2003 with a degree in chemical engineering. He obtained his Master's degree in 2006 from the East China University of Science and Technology, and his PhD in biological sciences in 2010 from Hokkaido University in Japan under the supervision of Jian Ping Gong. His PhD work focused on the self-assembling structures of lyotropic liquid crystals in solution and hydrogels. He is now with the group of Eugenia Kumacheva as a postdoctoral fellow at the University of Toronto in Canada.

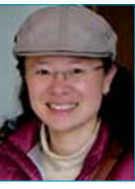

\section{Jian Ping Gong}

Jian Ping Gong received her Bachelor's degree in electronic physics from Zhejiang University in China, and her PhD for research on hightemperature superconductors in 1993 from the Tokyo Institute of Technology in Japan. She joined the Faculty of Science at Hokkaido University in 1993. She has been focusing on the fundamentals and functions of soft and wet materials, such as surface friction, toughening and fracture of gels. She has received the Wiley Polymer Science Award for her study of surface friction of polymer gels, and the Award of the Society of Polymer Science, Japan for developing double-network gels. 